\title{
Enhanced detection of atrial arrhythmias by implantable monitors: Have we found what we are looking for?
}

\author{
Michael Lloyd ${ }^{1}$ \\ ${ }^{1}$ Emory University
}

July 12, 2021

\section{Enhanced detection of atrial arrhythmias by implantable monitors: Have we found what we are looking for?}

Michael S. Lloyd

Emory University Hospital, Atlanta, GA

Funding: None

Disclosures: none

Address for correspondence:

1364 Clifton Road NE

Suite F424

Atlanta, GA 30322

Mlloyd2@emory.edu

"When you look at the dark side, careful you must be. For the dark side looks back."

Yoda

Yoda's admonishment is a variation on the adage, "be careful what you're looking for, because you just may find it". Modern implantable devices can overwhelm us with incidental arrhythmias of all types and barrage us with false reports of atrial fibrillation. The ideal implantable loop recorder would uncover a whole new "dark side" of atrial arrhythmias (AF/AT) that would have heretofore gone unrecognized, but also reject false-positive detections which are the major drawback of current technology.

In this edition of JCE[1], Saha and coworkers study an AF/AT detection algorithm designed for implantable loop recorders which is purported to go beyond the current state of the art in several ways: 1. A dual-stage method (detect/verify) with adaptive morphology is meant to appropriately distinguish true AF from other sources of irregularity, namely, ectopy. 2. It is enabled with a proprietary noise identification feature adapted from subcutaneous ICD technology that enhances noise rejection. 3. More regular atrial tachycardias/flutters may also be detected by an AT detection feature, which is an additional, separately programmable, rate detection process. 4. Finally, the device can be remotely programmed, which allows for adjustment of detection parameters based on real time analysis of detected episodes that were not true AF/AT. The parameters are tunable, with options for duration, sensitivity response (least, less, balanced, more, or most), and morphology assessment (on/off).

They subjected the algorithm to digital signals from holters and post-processed signals in the V2-V3 vector 12 lead EKGs from a large databank (THEW, University of Rochester). Each dataset contained a clinically 
adjudicated determination of the presence of $\mathrm{AT} / \mathrm{AF}$ and burden which was the gold standard. The dataset consisted of 1966 patients with a $3.9 \%$ prevalence of AF/AT of at least 4 minutes in duration.

They found that with nominal settings (4-minute detection window, "more response"), the algorithm detected $100 \%$ (76 or 76 ) of the true AF episodes. It falsely reported AF in an additional 16 patients for a positive predictive value of $82.6 \%$. It correctly identified 1874 of the 1890 patients without any AF to give a negative predictive value of $99.2 \%$ and a reported overall accuracy of $99.2 \%$. The authors noted that, without morphology and noise rejection features, the positive predictive value for AF burden would have fallen $12 \%$.

Of the 16 patients falsely reported to have AF, simulated remote programming changes (from "more response" to "less response" setting) resolved 12 patients' false detections.

The AT detection feature performed poorly when programmed at 2-minute durations (which is the setting for all other currently available monitors) but did well when lengthened to the 60-minute detection duration. However, the only events analyzed were atrial flutter of at least 3 hours in this dataset.

The findings re-ignite a smoldering question that underlies all studies of AT/AF detection devices: What burden or length of AT/AF truly matters? Analysis from Mode Selection Trial (MOST) data showed an adjusted HR of 2.79 for stroke or death among patients with 5-minute or longer episode of atrial arrhythmia, while the ASSERT trial showed a lower but significant risk for AF events over 6 minutes (HR 1.76). [2, 3] A large VA cohort showed the greatest benefit of anticoagulation was for AF episodes $>24$ hours. [4] Our group has shown that incidental detection of atrial fibrillation by implantable devices vary widely over time, so that even 30 seconds of atrial fibrillation (below the threshold of all currently available implantable monitors including the one studied here) may portend 2 hours of atrial fibrillation in the future.[5] The data thus far signal that a "dose-response" effect of AF/AT does probably exist. However, we will never know exactly how to deal with device-detected AT/AF until we better refine and understand the magnitude of the problem, which is exactly the purpose of the algorithm under study by Saha and coworkers.

There are limitations of the analysis that should be mentioned. Most of the authors have major conflicts of interest as they are employed by the company that sells the product. Digital data from surface electrocardiograms may not accurately represent the data recorded from the implantable monitor. The algorithm was not directly compared to other available algorithms so there was no control. Consequently, scientifically robust claims of superiority to the current state of the art cannot be made. Finally, as the authors acknowledge, in silico studies are far removed from in vivo data.

Despite these shortcomings, this work represents an important step towards enhanced detection of AT/AF, which better defines clinically actionable events to improve patients' lives. We eagerly await in vivo validation of this work and a comparative trial of this algorithm to current technology. If we have found what we are looking for, it is high time for studies focusing on precisely what to do with the information.

1. manuscript in press Saha et al.

2. Glotzer, T.V., et al., Atrial high rate episodes detected by pacemaker diagnostics predict death and stroke: report of the Atrial Diagnostics Ancillary Study of the MOde Selection Trial (MOST).Circulation, 2003. 107 (12): p. 1614-9.

3. Healey, J.S., et al., Subclinical atrial fibrillation and the risk of stroke. N Engl J Med, 2012. 366 (2): p. $120-9$.

4. Perino, A.C., et al., Practice Variation in Anticoagulation Prescription and Outcomes After DeviceDetected Atrial Fibrillation.Circulation, 2019. 139 (22): p. 2502-2512.

5. Al-Gibbawi, M., et al., Relationship between device-detected burden and duration of atrial fibrillation and risk of ischemic stroke.Heart Rhythm, 2021. 18 (3): p. 338-346. 